\title{
Geleitwort des „Deutschen Tourismusverbands“ (DTV)
}

\author{
Liebe Leserin, lieber Leser,
}

wie der Deutschlandtourismus insgesamt, steht auch der Kulturtourismus in Deutschland im Zeichen von Veränderung. Über lange Zeit galt die Gleichung Kulturtourismus = Städtetourismus. Mittlerweile hat er aber eine Vielzahl von Gesichtern ausgeprägt - städtische, ländliche, grüne oder kulinarische. So weist der GfK DestinationMonitor 2018 noch den Besuch kultureller Sehenswürdigkeiten als wichtigste Aktivität der deutschen Gäste im Urlaub aus. Wenn wir aber gleichzeitig sehen, dass der Aufenthalt in der Natur für Urlauber eine ebenso hohe Bedeutung gewinnt, lädt dies zum Weiterdenken und Weiterarbeiten ein. Vielleicht kann die Corona-Krise, die den Tourismus und die Kultur gleichermaßen schwer getroffen hat, hier sogar noch zu einem Katalysator werden.

Auch deswegen verfolgte der DTV von 2015 bis 2019 gemeinsam mit zahlreichen Partnern das Projekt „Die Destination als Bühne“, in dem Tourismusregionen und Akteure aus dem Kulturbereich gemeinsam Strategien für den Erfolg des Kulturtourismus im ländlichen Raum erarbeiteten. Auch hier zeigte sich: Veränderung bestimmt den Alltag. Nicht nur, dass Kulturtourismus den Weg Richtung ländliche Regionen eingeschlagen hat, auch die Anforderungen an die Kulturverantwortlichen und Tourismusorganisationen (DMOs) verändern sich rasant. Gerade im ländlichen Raum kann der Tourismus das kulturelle Angebot für die Einheimischen sichern. Wenn wir über Veranstaltungen als Reiseanlass hinausdenken, können - mit zeitgemäßen Konzepten beispielsweise Schlösser, Guts- und Herrenhäuser sowie Gartenanlagen und Parks ihr Potenzial und ihre Anziehungskraft voll ausspielen. So kann ein produktives Zusammenspiel in strukturschwachen Regionen zum Motor für Einkommen, Beschäftigung und nachhaltige Entwicklung werden. Dafür müssen allerdings DMOs ebenso wie Kulturschaffende den Blick über den eigenen Tellerrand hinaus riskieren, zusammenarbeiten und Bündnisse schmieden.

Erfolgversprechende Best-Practice-Beispiele entstehen bereits überall in Deutschland. Deswegen bemüht sich der DTV auch um eine neue Grundlagenuntersuchung des Bundes für diesen Bereich. Die letzte stammt aus dem Jahr 2006 und fokussierte naturgemäß noch auf das Zusammenspiel von Städte- und Kulturtourismus. Gerade zu einem Zeitpunkt, wo die Bundesregierung eine nationale Tourismusstrategie erarbeitet, wünschen wir uns, dass dieses für Deutschland wichtige Thema Kultur sehr viel mehr Berücksichtigung darin findet.

Das große Potenzial des Kulturtourismus kann nur durch gemeinsame Anstrengungen gehoben werden. Am größten ist die Aussicht auf Erfolg, wenn die Rollen klar verteilt sind: eine DMO, die sich als Moderator, als Dirigent begreift, und aktive Kulturschaffende, die es verstehen, überregionale Leuchttürme und auch kleinere Angebote 
gut miteinander zu vernetzen. Als DTV setzen wir uns seit Jahren für ein konstruktives Miteinander ein - zwischen den unterschiedlichen politischen Ebenen, zwischen den Verbänden und genauso auch zwischen den Tourismus- und Kulturpartnern. Weil wir mit gebündelten Interessen und gebündeltem Know-how viel mehr erreichen können, als viele kleine Initiativen nebeneinander das vermögen, führen Kooperation und Vernetzung zu höheren Erfolgsaussichten.

Bei allem Enthusiasmus und Potenzial muss uns klar sein: Eine solche Veränderung geht nicht von heute auf morgen. Sie braucht Zeit, Geduld und am besten von vorneherein eine langfristige Perspektive. Aber vielleicht kann gerade hier der Tourismus am meisten von der Kultur profitieren. Denn wie sagte schon Hippokrates: „Das Leben ist kurz, die Kunst währet lange.“

In diesem Sinn wünschen wir Ihnen eine spannende Lektüre und viele neue Erkenntnisse mit diesem lange überfälligen Grundlagenwerk zum Kulturtourismus.

Reinhard Meyer

Deutscher Tourismusverband e. V. Präsident
Norbert Kunz

Deutscher Tourismusverband e. V. Geschäftsführer 